\title{
Magnetic states in low-pinning high-anisotropy material nanostructures suitable for dynamic imaging
}

\author{
Felix Büttner, ${ }^{1,2,3}$ C. Moutafis, ${ }^{3,4, *}$ A. Bisig, ${ }^{1,3,5,7}$ P. Wohlhüter, ${ }^{5}$ C. M. Günther, ${ }^{2}$ J. Mohanty, ${ }^{2, \dagger}$ J. Geilhufe,${ }^{6}$ M. Schneider, ${ }^{2}$ \\ C. v. Korff Schmising, ${ }^{2}$ S. Schaffert, ${ }^{2}$ B. Pfau, ${ }^{2}$ M. Hantschmann, ${ }^{2}$ M. Riemeier, ${ }^{2}$ M. Emmel, ${ }^{1}$ S. Finizio, ${ }^{1}$ G. Jakob, ${ }^{1}$ \\ M. Weigand, ${ }^{7}$ J. Rhensius, ${ }^{5,8}$ J. H. Franken, ${ }^{9}$ R. Lavrijsen, ${ }^{9}$ H. J. M. Swagten, ${ }^{9}$ H. Stoll, ${ }^{7}$ S. Eisebitt, ${ }^{2,6}$ and M. Kläui ${ }^{1,3,5}$ \\ ${ }^{1}$ Institut für Physik, Johannes Gutenberg-Universität Mainz, 55099 Mainz, Germany \\ ${ }^{2}$ Institut für Optik und Atomare Physik, Technische Universität Berlin, Straße des 17. Juni 135, 10623 Berlin, Germany \\ ${ }^{3}$ SwissFEL, Paul Scherrer Institut, 5232 Villigen PSI, Switzerland and Laboratory for Nanomagnetism and Spin Dynamics, \\ École Polytechnique Fédérale de Lausanne, 1015 Lausanne, Switzerland \\ ${ }^{4}$ Swiss Light Source, Paul Scherrer Institut, 5232 Villigen PSI, Switzerland \\ ${ }^{5}$ Fachbereich Physik, University of Konstanz, 78457 Konstanz, Germany \\ ${ }^{6}$ Helmholtz-Zentrum Berlin für Materialien und Energie GmbH, Hahn-Meitner-Platz 1, 14109 Berlin, Germany \\ ${ }^{7}$ Max Planck Institute for Intelligent Systems, 70569 Stuttgart, Germany \\ ${ }^{8}$ Laboratory for Micro- and Nanotechnology, Paul Scherrer Institut, 5232 Villigen PSI, Switzerland \\ ${ }^{9}$ Department of Applied Physics, Center for NanoMaterials, Eindhoven University of Technology, \\ P.O. Box 513, 5600 MB Eindhoven, The Netherlands
}

(Received 2 November 2012; revised manuscript received 18 February 2013; published 26 April 2013)

\begin{abstract}
We present magnetic domain states in a material configuration with high (perpendicular) magnetic anisotropy and particularly low magnetic pinning. This material, a B-doped $\mathrm{Co} / \mathrm{Pt}$ multilayer configuration, exhibits a strong magnetic contrast in x-ray transmission experiments, making it apt for dynamic imaging with modern synchrotron techniques, providing high spatial and high temporal resolution simultaneously. By analyzing the static spin structures in nanodisks at variable external fields, we show that CoB/Pt multilayers exhibit low enough domain wall pinning to manipulate the domain pattern with weak stimuli and in particular to move domains and domain walls. We demonstrate in a proof-of-principle experiment using pump-probe x-ray holographic imaging that moderate magnetic fields can induce elastic and deterministic and hence repeatable small variations of the domain configuration in $\mathrm{CoB} / \mathrm{Pt}$ multilayers, which is the key to perform high-resolution imaging of the domain wall motion to gain insight to the details of the local magnetization dynamics.
\end{abstract}

DOI: 10.1103/PhysRevB.87.134422

PACS number(s): 75.75.-c, 75.50.Kj, 75.60.Ch, 75.78.Fg

\section{INTRODUCTION}

Materials with perpendicular magnetic anisotropy (PMA) are attracting significant scientific interest, motivated in particular by the technological relevance of this class of materials for applications in high-bit-density hard disk storage devices, ${ }^{1}$ current-driven domain wall shift registers with low critical current densities, ${ }^{2}$ and programmable magnetic logic elements. ${ }^{3}$ All these applications rely on the magnetic switching or controlled motion of magnetic domain walls at the nanometer length scale and picosecond time scale. The ongoing debate regarding the underlying physics of the domain dynamics in PMA systems, such as the origin, universality, magnitude, and even the $\operatorname{sign}^{4,5}$ of the nonadiabatic spin transfer torque, as well as the eigenmodes of confined magnetic bubbles $^{6}$ (bubbles can be viewed as the out-of-plane analog to the magnetic vortex $\left.{ }^{7,8}\right)$, underline the need for direct imaging of the domain dynamics at the relevant sub-50 $\mathrm{nm}$ length scale and subnanosecond time scale. Such high-resolution pump-probe dynamic imaging is well established for in-plane materials, in particular permalloy, with prominent experiments on, for instance, gyrating magnetic vortices ${ }^{9-12}$ and domain wall motion. ${ }^{13,14}$ For PMA materials, however, small relative variations in the sample structure are amplified by the high perpendicular anisotropy to form considerable energy barriers for magnetic domain wall motion (pinning sites). The influence of pinning is twofold and complementary on different length scales. While domain wall motion within a pinning potential is deterministic and elastic due to the well-defined restoring force of the potential, on length scales extending several pinning potential minima, stochastic thermal hopping over the energy barrier prevents elastic and in particular deterministic domain wall motion at small excitations and finite temperatures. That is, spin dynamics in high pinning materials is deterministic only at very small displacements (motion within the pinning potential) and in spin switching experiments at very high excitations (as strong pinning causes major hysteresis loop memory and reproducibility). In the latter case, changes are permanent (not elastic), and repeatability can be achieved only by a second pulse flipping back the spins and restoring the original configuration. To image domain wall motion, on the other hand, elasticity is needed on a length scale significantly larger than the spatial resolution, and thus shallow and sparsely distributed pinning sites are desirable. It has been demonstrated recently that pump-probe imaging of creep domain wall motion is possible, provided the domain wall can be reset to a well-defined starting position after every excitation, which, for instance, can be realized by using an artificial energy barrier. ${ }^{15}$ However, the stochastic motion during the excitation effectively reduces the time resolution, leading to uncertainties in the nanosecond regime. For numerous applications and basic science studies, e.g., for the observation of gyrating bubbles, it is desirable that the domain wall motion is deterministic on the subnanosecond time scale, and elastic, i.e., that the system returns to the initial configuration when no external stimulus 
is present. The availability of a suitable, low-pinning PMA material system is thus pivotal in this context.

Here we are using $\mathrm{x}$-ray imaging to determine the magnetic states. This is so far the only approach combining high spatial and high temporal resolution that allows for the imaging of magnetic domain dynamics in a pump-probe scheme. $\mathrm{X}$-ray holographic imaging ${ }^{16}$ in a transmission geometry is ideally suited as this technique is intrinsically drift free and hence allows for the reliable detection of changes in the domain pattern even down to a few nanometers. However, $\mathrm{x}$-ray-transmission-based dynamic imaging entails several constraints to the sample under investigation. First, the total thickness of the magnetic material has to be sufficient to provide reasonable magnetic contrast through the x-ray magnetic circular dichroism (XMCD) ${ }^{17}$ Specifically, imaging in a transmission geometry currently requires several nanometers of magnetic material. Materials with PMA, which can be prepared with the desired thickness on amorphous $\mathrm{Si}_{3} \mathrm{~N}_{4}$ membranes, are, for instance, multilayers of transition metals, such as $\mathrm{Co} / \mathrm{Pt}$ or $\mathrm{Co} / \mathrm{Pd}$. These materials have been commonly used in a wide range of experiments ${ }^{1,3,18-22}$ and are very well characterized. ${ }^{23-29}$ Second, the pump-probe scheme relies on a deterministic and elastic response of the magnetic domains and domain walls to drive forces of up to $\mathrm{GHz}$ repetition rates. This requires a material with particularly low pinning to provide the high repeatability of the domain wall motion, which is also of key importance for applications.

In this paper we present a perpendicular magnetic material configuration for deterministic domain wall motion based on $\mathrm{B}$-doped $\mathrm{Co} / \mathrm{Pt}$ multilayers. In this material a significant reduction of the domain wall pinning has been found using quasistatic measurement techniques on films with subnanometer Co thickness. ${ }^{30,31}$ For x-ray-transmission-based imaging, however, we need a minimum total thickness of a few nanometers of magnetic material. As the PMA in nonepitaxial $\mathrm{Co} / \mathrm{Pt}$ arises from the $\mathrm{Co} / \mathrm{Pt}$ interface interaction, ${ }^{32}$ a natural way to retain the magnetic properties in a few nanometers thick configuration is to use multilayer stacks. Using $\mathrm{x}$-ray holographic imaging of nanopatterned disks under statically applied external magnetic fields, we show that the pinning strength in our developed $\mathrm{CoB} / \mathrm{Pt}$ multilayer configuration is sufficiently low and can enable repetitive pump-probe dynamic imaging. We demonstrate the repeatability in a pump-probe experiment, showing that we can use the material to image domain dynamics with sub-50 $\mathrm{nm}$ spatial resolution and subnanosecond time resolution.

\section{EXPERIMENT}

Our B-doped magnetic multilayer is a $\mathrm{Ta}(2) / \mathrm{Pt}(2) /$ $\left[\mathrm{Co}_{68} \mathrm{~B}_{32}(0.35) / \mathrm{Pt}(0.7)\right]_{40} / \mathrm{Pt}(1.3)$ (thicknesses given in $\mathrm{nm}$ ) stack grown on a $\mathrm{Si}_{3} \mathrm{~N}_{4}$ membrane where a holographic mask with an $800 \mathrm{~nm}$ diameter object hole has been prepared by focused ion beam milling in a $1.5 \mu \mathrm{m}$ thick gold film on the back side before deposition. The magnetic film was grown using DC magnetron sputtering at a chamber base pressure of $3 \times 10^{-8}$ mbar. The $\mathrm{Co}_{68} \mathrm{~B}_{32}$ layers were grown at an Ar pressure of $1 \times 10^{-2} \mathrm{mbar}$ and a DC sputtering power of $20 \mathrm{~W}$, while for the Pt layers an Ar pressure of $3 \times 10^{-3}$ mbar at $60 \mathrm{~W}$ was used. The film was patterned after deposition into a $750 \mathrm{~nm}$ diameter disk centered to the object hole using electron beam lithography (EBL) and Ar ion milling. Finally, a $65 \mathrm{~nm}$ diameter reference hole was prepared adjacent to the object hole, yielding a resolution of $45(10) \mathrm{nm}^{33}$ To compare the magnetic properties, we investigate nanostructures of a representative transition metal multilayer stack: $\operatorname{Ta}(3) / \mathrm{Pd}(3) /[\mathrm{Co}(0.3) / \mathrm{Pd}(0.9)]_{50} / \mathrm{Pd}(1.1)$. The material is grown on a $\mathrm{Si}_{3} \mathrm{~N}_{4}$ membrane and patterned into a $600 \mathrm{~nm}$ diameter disk by EBL and lift-off. The holographic mask is fabricated on a separate membrane to define a $2 \mu \mathrm{m}$ object hole and an adjacent $50 \mathrm{~nm}$ reference hole in a $900 \mathrm{~nm}$ thick gold film, yielding a resolution of approximately $35(10) \mathrm{nm}$. The mask is aligned to the magnetic disk using piezo stages. ${ }^{34}$ This approach requires a special image reconstruction procedure to achieve optimal spatial resolution due to the finite distance between sample and mask $\left(11 \mu \mathrm{m}\right.$ in our case) ${ }^{35}$ The holographic imaging was performed at the UE-52 SGM beamline at the BESSY II synchrotron in Berlin using circularly polarized photons with energy tuned to the Co $\mathrm{L}_{3}$ absorption edge (778 eV), employing XMCD to obtain magnetic contrast.

From superconducting quantum interference device (SQUID) measurements on a continuous $\mathrm{CoB} / \mathrm{Pt}$ multilayer film with the exact same composition, we obtain a saturation magnetization of $M_{s}=1.24(3) \times 10^{6} \mathrm{~A} / \mathrm{m}$ (when normalized to the total thickness of the magnetic $\mathrm{CoB}$ ), and a hard axis saturation field of $\mu_{0} H_{k}=0.34(5) \mathrm{T}$ corresponding to a total (effective) uniaxial magnetic anisotropy of $K_{u, \text { eff }}=2.1(3) \times 10^{5} \mathrm{~J} / \mathrm{m}^{3}$. Here $K_{u, \text { eff }}>0$ defines a preferred out-of-plane alignment of magnetic moments. In a single-layer configuration $\left(\mathrm{Pt}(4) / \mathrm{Co}_{68} \mathrm{~B}_{32}(0.6) / \mathrm{Pt}(2)\right)$, similar values of $M_{s}=1.03 \times 10^{6} \mathrm{~A} / \mathrm{m}$ and $K_{u, \text { eff }}=3.2 \times 10^{5} \mathrm{~J} / \mathrm{m}^{3}$ have been found before. ${ }^{30,31}$ To calculate the crystal field uniaxial anisotropy constant $K_{u}$, we make use of the analytic relation $\mathbf{H}=\frac{\partial F}{\mu_{0} \partial \mathbf{M}}$ between the magnetic field $\mathbf{H}$ and the magnetization $\mathbf{M}$ through the free energy density $F$, which holds if the system is in thermodynamic equilibrium. ${ }^{36} \mathrm{In}$ absence of any further knowledge of the magnetization reversal mechanism, the equilibrium magnetization can be approximated by the average of both hysteresis branches, with the difference giving a reasonable estimate for the $2 \sigma$ confidence interval. The free energy density is a potential, for which we can hence choose a constant offset such that the free energy density of the in-plane saturated state is $F_{s, \text { ip }}=0$. Bringing the system from in-plane to out-of-plane saturation costs demagnetization energy and yields anisotropy energy. The free energy density of the out-of-plane saturated state is hence $F_{s, \text { oop }}=\mu_{0} M_{s}^{2} / 2-K_{u}$. The remanent (demagnetized) state shows complex domains, which makes the corresponding free energy density $F_{0}$ difficult to calculate. Here, however, we only use that the value $F_{0}$ is unique and therefore equal for the in-plane and the out-of-plane loop. The energy differences $F_{s, \text { oop }}-F_{0}$ and $F_{s, \text { ip }}-F_{0}$ can be determined by calculating the saturation energies $E_{s, \text { oop }}$ and $E_{s, \text { ip }}$, respectively, from the measured hysteresis loops ${ }^{36}$ Combining these considerations, we arrive at $K_{u}=\mu_{0} M_{s}^{2} / 2-E_{\mathrm{s}, \mathrm{oop}}+E_{\mathrm{s}, \mathrm{ip}}$. For our material configuration, we obtain a value of $K_{u}=$ $1.01(5) \times 10^{6} \mathrm{~J} / \mathrm{m}^{3}$, corresponding to a quality factor of $Q=$ $1.05(8)$. With these properties, our material is comparable to typical $\mathrm{Co} / \mathrm{Pt}$ or $\mathrm{Co} / \mathrm{Pd}$ multilayers, ${ }^{23-28}$ and in contrast to other 

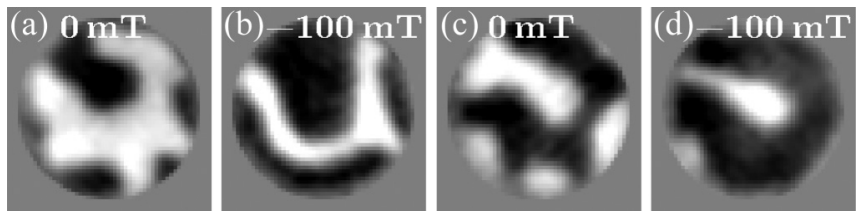

FIG. 1. Evolution of the magnetic domain pattern in a $600 \mathrm{~nm}$ diameter $\mathrm{Co} / \mathrm{Pd}$ disk during application of a uniform static external field of varying strength. White indicates magnetization pointing up (positive field direction) and black indicates magnetization pointing down. Between (b) and (c), the sample was saturated with a magnetic field of $-200 \mathrm{mT}$.

low-pinning materials such as permalloy, it belongs to the class of high-anisotropy materials.

\section{STATIC PROPERTIES OF Co/Pd}

In Fig. 1 we present static magnetic images of a $\mathrm{Co} / \mathrm{Pd}$ disk at various uniformly applied external magnetic fields, and based on them we analyze the magnetic behavior of nanostructures from this material. Due to the inhomogeneity of the field and the hysteresis of the electromagnet, the field values given in the following are approximate, with an error of $6 \mathrm{mT}+10 \%$ of the actual value. We first image the domain configuration at zero applied field in Fig. 1(a). Subsequently, a downward-oriented magnetic field of $100 \mathrm{mT}$ is applied, see Fig. 1(b). After apparent saturation at $200 \mathrm{mT}$ (not shown), the field is reduced to zero again [Fig. 1(c)]. Immediately afterwards $100 \mathrm{mT}$ are applied in the downwards direction again, see Fig. 1(d), where a magnetic bubblelike domain is isolated. ${ }^{37}$ From the imbalance of black and white domains at zero field, we can deduce a considerable remanent magnetization of the sample. The observed domains show, both at remanence and at applied fields, a large variation in width and shape. Moreover, the shape of the domains is not commensurate with the disk geometry. The irregularity is present at the edges of the disk as well as in the center. Note that the spin configuration in Fig. 1(c) largely resembles that in Fig. 1(a), only with inverted contrast signaling the reversal of the magnetic moments. This reversal of magnetic moments as well as the substantially different intermediate state in Fig. 1(b) excludes insufficient saturation as an explanation for the similarity (as argued in Ref. 23). Rather, our observations point to the presence of considerable pinning sites distributed all over the sample. The pinning is usually attributed to local variations in the anisotropy. It has been reported in Refs. 1 and 26 that this variation could be explained by the different orientation of crystal grains in the polycrystalline Co. The presence of strong pinning sites as found in our $\mathrm{Co} / \mathrm{Pd}$ material is highly desirable for applications in data storage devices to stabilize the domain configuration against external perturbations, and the reproducibility of the remanent domain configuration could be used to investigate, for instance, the switching dynamics induced by very strong excitations. For the specific purpose of pump-probe dynamic imaging of domain wall motion, however, a system with significantly weaker pinning centers and a reduced pinning density is required.
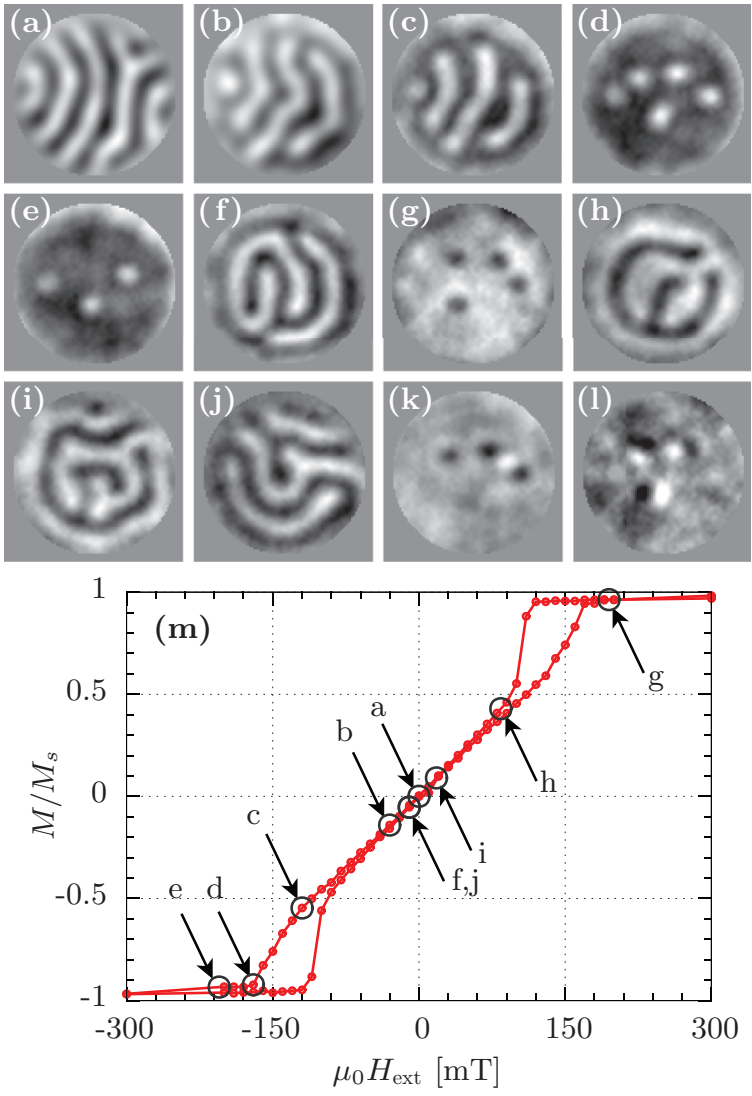

FIG. 2. (Color online) (a)-(1) Selection of magnetization images of a $750 \mathrm{~nm}$ diameter $\mathrm{CoB} / \mathrm{Pt}$ disk at various out-of-plane magnetic field steps, where white indicates magnetization pointing up (positive field direction) and black indicates magnetization pointing down. (a) shows the virgin state after deposition. Between (a) and (b), the sample experienced a field of $65 \mathrm{mT}$. Between (e) and (f), between (g) and (h), and between (i) and (j), the sample was saturated. (k) visualizes the difference of (d) and (e), and (l) depicts the sum of (d) and (g), highlighting the different positions of the bubbles in these images. (m) shows an out-of-plane hysteresis loop of a continuous $\mathrm{CoB} / \mathrm{Pt}$ film, in which the field values corresponding to images (a)-(1) are illustrated.

\section{STATIC PROPERTIES OF CoB/Pt}

In Fig. 2 we present an out-of-plane hysteresis loop of a continuous $\mathrm{CoB} / \mathrm{Pt}$ film together with a selection of images of a $750 \mathrm{~nm}$ diameter $\mathrm{CoB} / \mathrm{Pt}$ multilayer disk at relevant field values of a similar hysteresis loop. In Fig. 2(a) we see the virgin state after deposition. This remanent state shows, to the limit of our spatial resolution, equally distributed black and white domains as well as smooth domain walls. The domains with an average width of $68(4) \mathrm{nm}$ are clearly resolved. Between Figs. 2(a) and 2(b), the disk experienced a field of $65 \mathrm{mT}$, causing a significant increase of the average domain width to $83(5) \mathrm{nm}$. This is the domain width of the minority domains in all subsequent images. Comparing Figs. 2(b) and 2(c) we notice that an applied field of up to $120 \mathrm{mT}$ changes the domain configuration gradually by a continuous retraction of the unfavorable domains from the edge of the disk, leaving the domain arrangement and the widths of both black and white domains in the center approximately constant. When further 
increasing the field, the domains contract to multiple bubbles, see Fig. 2(d). The diameter of these bubbles equals, to the limit of our resolution, the widths of the minority domains that we find in any stripe-domain pattern along the hysteresis loop. In this part of the hysteresis loop, which corresponds to the only hysteretic part of the macroscopic $M-H$ loop, the magnetic field required to induce sizable changes in the domain configuration is of the order of a few tens of $\mathrm{mT}$ only. A further increase of the field reduces the number of bubbles, and one of the remaining bubbles moves to restore an energetically more favorable configuration, see Figs. 2(e) and 2(k), the latter showing the differences between Figs. 2(d) and 2(e) to highlight the new position of one of the bubbles. After saturation and returning close to remanence [Fig. 2(f)], we find a highly symmetric, meanderlike domain configuration with equally distributed black and white domains and smooth domain walls as before in Fig. 2(a). When applying the external field in the opposite direction, we observe, similar to images Figs. 2(d) and 2(e), multibubble states before saturation of the disk, but now at entirely different positions, c.f. Fig. 2(g). This is also visualized in the sum of Figs. 2(d) and 2(g) shown in Fig. 2(1). After saturation, we observe in particular on the descending branch of the hysteresis loop [Fig. 2(h)], but also at remanence [Fig. 2(i)], once more highly symmetric states, which, however, are otherwise not correlated to the patterns we found before in Figs. 2(a) and 2(f). The formation of symmetric patterns without correlation to previous field cycles is a general property of the sample, which is demonstrated also in another remanent state in Fig. 2(j). Following the terminology used by Pierce et al. ${ }^{29}$ to characterize the (microscopic) hysteresis and the related pinning in $\mathrm{Co} / \mathrm{Pt}$-type materials, the presented data (consistent with all the images that we acquired on the system) demonstrate that our patterned $\mathrm{CoB} / \mathrm{Pt}$ material has neither major loop microscopic return point memory nor major loop microscopic complementary point memory.

The increase of the initial as-grown domain width to an apparent intrinsic width of 83(5) $\mathrm{nm}$ after applying a small external field can be related to the formation of a metastable configuration during fabrication, which included the heating of the sample for the lithography process. In general, our observations reveal a low density of pinning sites so that there are very few considerable energy barriers that separate different energetically degenerate magnetic states. This is particularly obvious from the consistently circular symmetry of the observed domain configurations commensurate with the circular geometry. From the absence of return point memory and complementary point memory, we conclude that the different energetically degenerate magnetic states that are commensurate with the symmetry of the geometry, such as those shown in Figs. 2(f) and 2(i), are accessible from the saturated state without significant energy barriers (which would favor just one state). This means the energy landscape is rather flat, as any roughness in this energy landscape, which defines the pinning barriers, is overcome by the shape anisotropy energy, which hence sets an upper limit for the average pinning strength. As a shape anisotropy results from in-plane components of the stray field, this energy is small. The smooth domain walls and the constant width of the minority domains are further indications for a particularly low pinning strength and density in our material configuration.
The fact that the observed bubbles are not located at predefined positions and that they can be moved by moderate field changes demonstrates additionally that in our material configuration sparsely distributed strong pinning sites are absent. Therefore, bubbles are not trapped in a considerable pinning potential well, which opens the possibility to observe some of the recently predicted dynamical behavior of magnetic bubbles arising from their topological properties. ${ }^{6}$

\section{DYNAMIC IMAGING IN CoB/Pt}

To demonstrate the suitability of the material for pumpprobe dynamic imaging of field-induced domain dynamics, a microcoil was fabricated lithographically around a disk of the $\mathrm{CoB} / \mathrm{Pt}$ material configuration. Through this microcoil, $4 \mathrm{~ns}$ long rectangularly shaped pulses were injected at a repetition rate of $1.25 \mathrm{MHz}$, generating an approximately uniform outof-plane field of $25 \mathrm{mT}$. The pulse injection was synchronized with the probing $\mathrm{x}$-rays to allow for imaging of the magnetic state at variable times after the start of the pulse. We set the system in a multibubble configuration by applying an in situ static magnetic field of $140 \mathrm{mT}$ parallel to the excitation field. To demonstrate the repeatability, we present an image of the magnetic state during the pulse and one image long after the pulse (46 ns), where each image has been reconstructed from the accumulated average of several $10^{9}$ pump-probe iterations. The total accumulation of the two images was subdivided into several sequences of alternating time delays to confirm that the observed differences are fully dynamical in nature and not due to a single, thermally activated event of domain wall motion.

In Fig. 3 we present dynamic images of the magnetic domain configuration long after the pulse in Fig. 3(b) and on the plateau of the pulse in Fig. 3(c). Both images show clearly different magnetic contrast. In both images, we can identify three domains [highlighted by black dotted lines in Figs. 3(b) and 3(c)] with magnetic moments pointing downwards, opposite to the upward-oriented external field and the otherwise upwards magnetized sample. When imaging
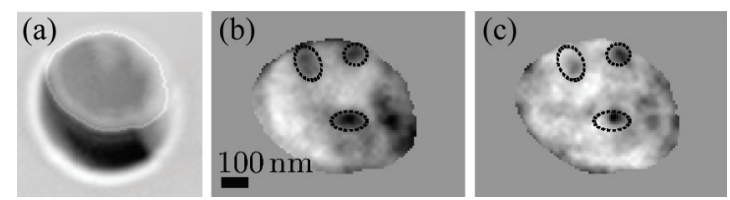

FIG. 3. Pump-probe dynamic imaging of the excitation of a multibubble magnetic configuration in a $700 \mathrm{~nm} \mathrm{CoB} / \mathrm{Pt}$ multilayer disk with a static bias field of $140 \mathrm{mT}$ in the out-of-plane direction. In (a) we see the topography of the sample, with the large circle defining the object hole in the opaque Au film. The area of magnetic material is highlighted by a lighter boundary and only the inner part is shown in (b) and (c). In (b) we show the magnetic state long after (46 ns) a $25 \mathrm{mT}$ homogeneous magnetic field pulse of $4 \mathrm{~ns}$ duration, where black indicates magnetization pointing down, white indicates magnetization pointing up (positive field direction). This corresponds to the state without a field pulse. The black dotted lines mark the borders of bubblelike configurations of down-pointing magnetic moments. (c) depicts the domain configuration of the disk on the plateau of the pulse, and the border of the bubble domains off the pulse is sketched again with dotted lines to highlight the shrinking of the bubbles. 
long after the pulse, we observe that these minority domains are enlarged and slightly elliptical. During the pulse [Fig. 3(c)], the minority domains shrink to a rounder shape, a dynamics that resembles a breathing mode of magnetic bubbles expected for homogeneous out-of-plane fields. To highlight the changes due to the field pulse, the border of the bubbles in the remanent state has been overlaid on both images with dotted lines. All three bubbles shrink when we apply this field pulse in the up direction. The shrinking and expansion is highly anisotropic. Only the long axes of the elliptical domains vary considerably. The length of the short axes remains constant with varying field strength, which is in agreement with our observation of a constant width of minority domains in the static hysteresis loop.

These proof-of-principle images of dynamic domain changes demonstrate that our material configuration is magnetically sufficiently soft to allow for inducing significant and clearly detectable changes using microcoil-generated nanosecond field pulses with several $\mathrm{MHz}$ repetition rate. The dynamic response of the system is deterministic and elastic, allowing us to obtain clear images from an accumulated average of several $10^{9}$ pump-probe iterations.

\section{CONCLUSION}

From the observed static domain states at variable external fields, we conclude that $\mathrm{CoB} / \mathrm{Pt}$ multilayers exhibit a very weak influence of pinning on the magnetic states. This leads to the necessary viscous motion of domain walls in an external magnetostatic potential and the resulting reproducibility for repetitive pump-probe imaging of magnetic domain dynamics. We demonstrate the suitability of this material in a dynamic pump-probe imaging experiment of multibubble soliton ${ }^{38}$ states, where we were able to observe and repeat the expansion of bubbles to elliptical domains and the shrinking back to bubble states. For high-anisotropy systems, our material configuration has the potential to become of equally widespread use as permalloy for low-anisotropy systems, and thereby enabling a comparable range of fundamental experiments for this technologically important class of materials.

\section{ACKNOWLEDGMENTS}

We thank C. Barton, C. Morrison, and T. Thomson for providing the $\mathrm{Co} / \mathrm{Pd}$ material. This work was funded by the German Ministry for Education and Science (BMBF), projects "Multimag" (13N9911) and "MPscatt" (05K10KTB), the Graduate School "Material Science in Mainz" (DFG/GSC 266), EU's 7th Framework Programme IFOX (NMP3-LA2010 246102), MAGWIRE (FP7-ICT-2009-5 257707), the European Research Council through the Starting Independent Researcher Grant MASPIC (ERC-2007-StG 208162), the Swiss National Science Foundation, and the DFG. *christoforos.moutafis@psi.ch

†Present address: Department of Physics, Indian Institute of Technology Hyderabad, Ordnance Factory Estate, Yeddumailaram, Andhra Pradesh 502205, India.

${ }^{1}$ B. Pfau, C. M. Günther, E. Guehrs, T. Hauet, H. Yang, L. Vinh, X. Xu, D. Yaney, R. Rick, S. Eisebitt, and O. Hellwig, Appl. Phys. Lett. 99, 062502 (2011).

${ }^{2}$ Y. Zhang, W. S. Zhao, D. Ravelosona, J.-O. Klein, J. V. Kim, and C. Chappert, J. Appl. Phys. 111, 093925 (2012).

${ }^{3}$ S. Breitkreutz, J. Kiermaier, S. Vijay Karthik, G. Csaba, D. Schmitt-Landsiedel, and M. Becherer, J. Appl. Phys. 111, 07A715 (2012).

${ }^{4}$ J. Heinen, D. Hinzke, O. Boulle, G. Malinowski, H. J. M. Swagten, B. Koopmans, C. Ulysse, G. Faini, B. Ocker, J. Wrona, and M. Kläui, J. Phys.: Condens. Matter 24, 024220 (2012).

${ }^{5}$ J. Xiao, A. Zangwill, and M. D. Stiles, Phys. Rev. B 73, 054428 (2006).

${ }^{6}$ C. Moutafis, S. Komineas, and J. A. C. Bland, Phys. Rev. B 79, 224429 (2009).

${ }^{7}$ C. Moutafis, S. Komineas, C. A. F. Vaz, J. A. C. Bland, and P. Eames, Phys. Rev. B 74, 214406 (2006).

${ }^{8}$ C. Moutafis, S. Komineas, C. A. F. Vaz, J. A. C. Bland, T. Shima, T. Seki, and K. Takanashi, Phys. Rev. B 76, 104426 (2007).

${ }^{9}$ K. Tanabe, D. Chiba, J. Ohe, S. Kasai, H. Kohno, S. E. Barnes, S. Maekawa, K. Kobayashi, and T. Ono, Nat. Commun. 3, 845 (2012).

${ }^{10}$ B. Van Waeyenberge, A. Puzic, H. Stoll, K. W. Chou, T. Tyliszczak, R. Hertel, M. Fahnle, H. Bruckl, K. Rott, G. Reiss, I. Neudecker, D. Weiss, C. H. Back, and G. Schutz, Nature (London) 444, 461 (2006).
${ }^{11}$ M. Kammerer, M. Weigand, M. Curcic, M. Noske, M. Sproll, A. Vansteenkiste, B. Van Waeyenberge, H. Stoll, G. Woltersdorf, C. H. Back, and G. Schuetz, Nat. Commun. 2, 279 (2011).

${ }^{12}$ A. Bisig, J. Rhensius, M. Kammerer, M. Curcic, H. Stoll, G. Schütz, B. Van Waeyenberge, K. W. Chou, T. Tyliszczak, L. J. Heyderman, S. Krzyk, A. von Bieren, and M. Kläui, Appl. Phys. Lett. 96, 152506 (2010).

${ }^{13}$ J. Rhensius, L. Heyne, D. Backes, S. Krzyk, L. J. Heyderman, L. Joly, F. Nolting, and M. Kläui, Phys. Rev. Lett. 104, 067201 (2010).

${ }^{14}$ L. Heyne, M. Kläui, D. Backes, T. A. Moore, S. Krzyk, U. Rüdiger, L. J. Heyderman, A. Fraile Rodríguez, F. Nolting, T. O. Mentes, M. Á. Niño, A. Locatelli, K. Kirsch, and R. Mattheis, Phys. Rev. Lett. 100, 066603 (2008).

${ }^{15}$ J. Vogel, M. Bonfim, N. Rougemaille, O. Boulle, I. M. Miron, S. Auffret, B. Rodmacq, G. Gaudin, J. C. Cezar, F. Sirotti, and S. Pizzini, Phys. Rev. Lett. 108, 247202 (2012).

${ }^{16}$ S. Eisebitt, J. Lüning, W. F. Schlotter, M. Lorgen, O. Hellwig, W. Eberhardt, and J. Stöhr, Nature (London) 432, 885 (2004).

${ }^{17}$ G. Schütz, W. Wagner, W. Wilhelm, P. Kienle, R. Zeller, R. Frahm, and G. Materlik, Phys. Rev. Lett. 58, 737 (1987).

${ }^{18}$ A. J. Schellekens, A. v. d. Brink, J. H. Franken, H. J. M. Swagten, and B. Koopmans, Nat. Commun. 3, 847 (2012).

${ }^{19}$ M. S. Pierce, R. G. Moore, L. B. Sorensen, S. D. Kevan, O. Hellwig, E. E. Fullerton, and J. B. Kortright, Phys. Rev. Lett. 90, 175502 (2003).

${ }^{20}$ G. Malinowski, F. Dalla Longa, J. H. H. Rietjens, P. V. Paluskar, R. Huijink, H. J. M. Swagten, and B. Koopmans, Nat. Phys. 4, 855 (2008). 
${ }^{21}$ J. Heinen, O. Boulle, K. Rousseau, G. Malinowski, M. Kläui, H. J. M. Swagten, B. Koopmans, C. Ulysse, and G. Faini, Appl. Phys. Lett. 96, 202510 (2010).

${ }^{22}$ C. M. Günther, F. Radu, A. Menzel, S. Eisebitt, W. F. Schlotter, R. Rick, J. Lüning, and O. Hellwig, Appl. Phys. Lett. 93, 072505 (2008).

${ }^{23}$ J. E. Davies, O. Hellwig, E. E. Fullerton, G. Denbeaux, J. B. Kortright, and K. Liu, Phys. Rev. B 70, 224434 (2004).

${ }^{24}$ D. G. Stinson and S.-C. Shin, J. Appl. Phys. 67, 4459 (1990).

${ }^{25}$ Z. Liu, R. Brandt, O. Hellwig, S. Florez, T. Thomson, B. Terris, and H. Schmidt, J. Magn. Magn. Mater. 323, 1623 (2011).

${ }^{26}$ T. Thomson, G. Hu, and B. D. Terris, Phys. Rev. Lett. 96, 257204 (2006).

${ }^{27}$ S. Hashimoto, Y. Ochiai, and K. Aso, J. Appl. Phys. 66, 4909 (1989).

${ }^{28}$ D. Smith, V. Parekh, C. E, S. Zhang, W. Donner, T. R. Lee, S. Khizroev, and D. Litvinov, J. Appl. Phys. 103, 023920 (2008).

${ }^{29}$ M. S. Pierce, C. R. Buechler, L. B. Sorensen, S. D. Kevan, E. A. Jagla, J. M. Deutsch, T. Mai, O. Narayan, J. E. Davies, K. Liu, G. T. Zimanyi, H. G. Katzgraber, O. Hellwig, E. E. Fullerton, P. Fischer, and J. B. Kortright, Phys. Rev. B 75, 144406 (2007).

${ }^{30}$ R. Lavrijsen, G. Malinowski, J. H. Franken, J. T. Kohlhepp, H. J. M. Swagten, B. Koopmans, M. Czapkiewicz, and T. Stobiecki, Appl. Phys. Lett. 96, 022501 (2010).
${ }^{31}$ R. Lavrijsen, M. A. Verheijen, B. Barcones, J. T. Kohlhepp, H. J. M. Swagten, and B. Koopmans, Appl. Phys. Lett. 98, 132502 (2011).

${ }^{32}$ B. N. Engel, C. D. England, R. A. Van Leeuwen, M. H. Wiedmann, and C. M. Falco, Phys. Rev. Lett. 67, 1910 (1991).

${ }^{33}$ W. F. Schlotter, Ph.D. Thesis, dissertation, Stanford University, 2007.

${ }^{34}$ D. Stickler, R. Frömter, H. Stillrich, C. Menk, C. Tieg, S. Streit-Nierobisch, M. Sprung, C. Gutt, L.-M. Stadler, O. Leupold, G. Grübel, and H. P. Oepen, Appl. Phys. Lett. 96, 042501 (2010).

${ }^{35}$ E. Guehrs, C. M. Günther, B. Pfau, T. Rander, S. Schaffert, W. F. Schlotter, and S. Eisebitt, Opt. Express 18, 18922 (2010).

${ }^{36}$ F. Büttner, K. Zhang, S. Seyffarth, T. Liese, H.-U. Krebs, C. A. F. Vaz, and H. Hofsäss, Phys. Rev. B 84, 064427 (2011).

${ }^{37}$ C. Moutafis, J. Rhensius, A. Bisig, F. Büttner, C. Barton, C. Morrison, T. Thomson, C. Tieg, S. Schaffert, B. Pfau, C. M. Günther, S. Eisebitt, and M. Kläui, in 2011 International Conference on Electromagnetics in Advanced Applications (ICEAA) (IEEE, Piscataway, NJ, 2011), pp. 1121-1123.

${ }^{38}$ S. T. Chui and V. N. Ryzhov, Phys. Rev. Lett. 78, 2224 (1997). 